\title{
Evaluation of the usefulness of the SAGES Safe Cholecystectomy Program from the viewpoint of the European surgeon
}

\author{
Paweł Bogacki ${ }^{1}$, Jan Krzak², Katarzyna Gotfryd-Bugajska ${ }^{3}$, Mirosław Szura ${ }^{1}$ \\ ${ }^{1}$ Department of Clinical and Experimental Surgery, Jagiellonian University Medical College, Krakow, Poland \\ ${ }^{2}$ Department of Surgery, South Jutland Hospital, Aabenraa, Denmark \\ ${ }^{3}$ Department of General and Oncological Surgery, St John Grande Hospital, Krakow, Poland
}

Videosurgery Miniinv 2020; 15 (1): 80-86 DOI: https://doi.org/10.5114/wiitm.2019.83297

\begin{abstract}
Introduction: Common bile duct injury (CBDI) is a severe complication of laparoscopic cholecystectomy (LC). To minimize its occurrence, SAGES established the Safe Cholecystectomy Program (SCP) with 6 rules to follow during surgery.

Aim: To assess the knowledge of SCP among European surgeons and their opinion on its usefulness.

Material and methods: Data were gathered using questionnaires during surgical conferences in Poland and Denmark. The questionnaire asked about the surgeon's experience in cholecystectomy and the number of complications in the form of CBDI. It asked about the surgeon's opinion on the usefulness of SCP rules on a 10-point scale. A comparison between specialists and residents was performed. The study has been registered in the ClinicalTrials. gov - NCT03155321.

Results: One hundred eighty-four questionnaires were gathered. One hundred fourteen (61.96\%) specialists (72.8\% male, mean age: 50 years) and 70 (38.04\%) residents (56\% male, mean age: 34 years) completed the questionnaire. Mean work experience was 22 years among specialists and 4.5 years among residents. A high percentage of specialists have experienced CBDI ( $46 \%$ vs. $17 \%$ of residents, $p=0.014)$. More specialists are familiar with the SCP than residents (49.3\% vs. 21.7\%, $p=0.021$ ). Significant differences in the mean usefulness score were observed for three rules: rules 2 and 6 were found more useful by residents (mean score: $7.07 \mathrm{vs.} 6.01, p=0.025$ and $8.70 \mathrm{vs} .8 .27, p<0.001$ ), and rule 3 was found more useful by specialists (mean: 8.73 vs. 8.36, $p<0.001$ ).

Conclusions: The awareness of the SCP in Europe is low. Participants consider the rules of the SCP to be useful during surgery, although there are differences in the usefulness scores between the groups. An educational program to promote and further implement the SCP should be established.
\end{abstract}

Key words: complications, safety, laparoscopic cholecystectomy, Safe Cholecystectomy Program, common bile duct injury.

\section{Introduction}

Laparoscopic cholecystectomy (LC) is one of the most common general surgical procedures performed each year [1]. The first LC was performed in
1985 and in the 1990s it quickly gained in popularity with its obvious advantages over open cholecystectomy (OC) it terms of wound infection, length of stay, sepsis or pneumonia [2]. However, it was soon noticed that LC is associated with higher risk of com-

\section{Address for correspondence}

Paweł Bogacki MD, Department of Clinical and Experimental Surgery, Jagiellonian University Medical College, 80 Prądnicka St,

31-202 Krakow, Poland, phone: +48 667177 677, e-mail: pawelbogacki1@gmail.com 
mon bile duct injury (CBDI) in comparison to OC. The data in the literature are inconsistent, but the prevalence of CBDI after LC is reported to be in the range of $0.1-2.4 \%$ of the cases. Given the commonality of the procedure, this poses a significant burden on patients, doctors and health care systems in terms of the sheer frequency of this dangerous and costly complication. It was believed that the initial high prevalence of CBDI after LC was associated with the learning curve for the new adopted technique; however, after the initial decade in which a reduction in the rate of CBDI was noted, a plateau was achieved and in the last 15 years not much has changed in this regard globally. However, there are hospitals that were able to further minimize the incidence of this complication by applying the Critical View of Safety (CVS) technique in identifying the critical anatomical structures during surgery [3]. Most recent publications report the incidence of CBDI in the CVS hospitals to be as low as $0.23 \%$ [4], and one study proved it possible to go beyond the learning curve and reported a $0.08 \%$ incidence of CBDI in a 156,958 patient cohort [5], equaling the level CBDI after OC. The Critical View of Safety was invented by Strasberg and first published in 1995. (Such an approach to ductal identification had been described in 1992, but the term Critical View of Safety was used first in Strasberg's 1995 article.) It describes a specific laparoscopic view to be achieved during surgery, which minimizes the risk of misidentifying tubular structures and thus causing bile duct injury (BDI). The
CVS suggests that first, the triangle of Calot should be cleared of fat and fibrous tissue. Than, the gallbladder should be dissected from the cystic plate starting from the triangle of Calot and going up towards the cystic fundus. The dissection should proceed as far towards the cystic fundus as to expose the cystic plate - usually one third of the gallbladder is enough. Thereafter, two and only two ductal structures should be seen entering the gallbladder. This should be visualized from both the frontal and the rear view. This completes the CVS and only then should the clipping and cutting of the structures be performed.

Having realized that in hospitals in which CVS was introduced, a reduction of BDI occurrence was achieved [6-8], the Society of American Gastrointestinal and Endoscopic Surgeons (SAGES) developed a Safe Cholecystectomy Program (SCP) introducing the Critical View of Safety and the Culture of Safety in Laparoscopic Cholecystectomy to further decrease the number of BDI. The rules of the SCP are described briefly below.

The first rule tells the surgeon to use CVS to identify the cystic duct and cystic artery. It gives clear instructions on how to achieve it: first, the hepatocystic triangle should be cleared of fat and fibrous tissue. Next, the lower one third of the gallbladder is separated from the liver to expose the cystic plate. Then, two and only two structures should be seen entering the gallbladder. See intraoperative pictures of CVS below (Figure 1).

\section{A}

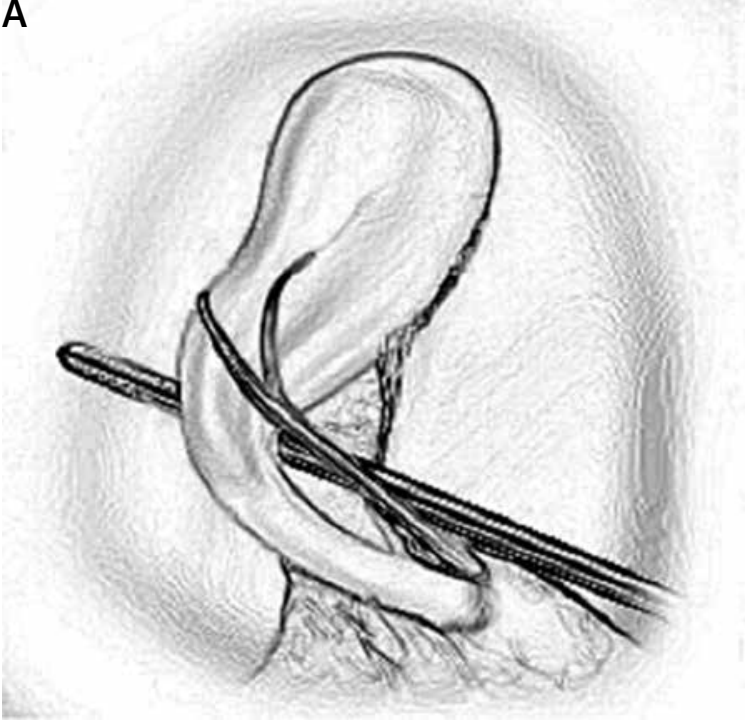

B

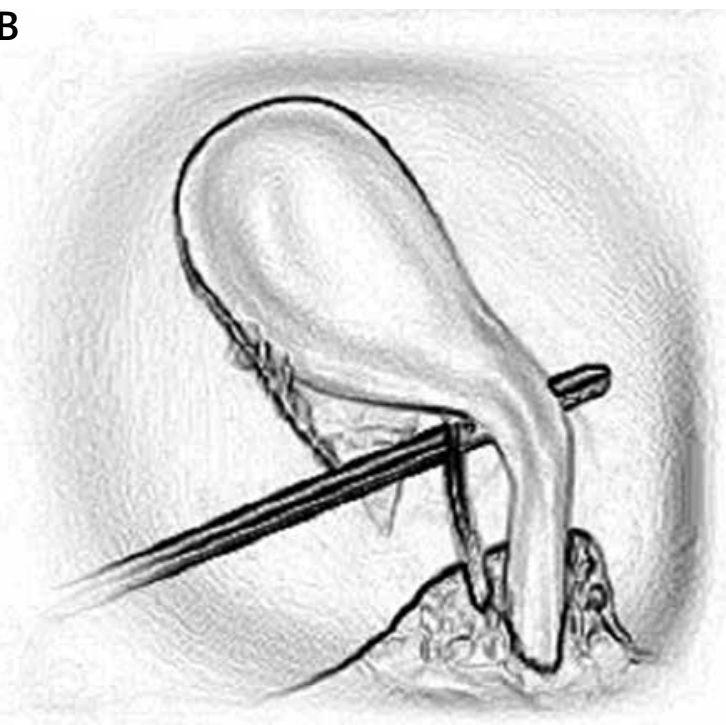

Figure 1. Critical view of safety: A - anterior view, B - posterior view 
The second rule tells us to consider intra-operative time-out prior to clipping, cutting of transecting any ductal structure. This is to confirm that the CVS has been correctly achieved.

The third rule reminds us to think about the potential for aberrant anatomy in all cases. That may be a short cystic duct, aberrant hepatic ducts or a right hepatic artery that crosses the anterior to the common bile duct.

Rule number 4 suggests the use of cholangiography or other methods to image the biliary tree intraoperatively if doubts about the anatomy occur.

Rule number 5 tells us to recognize a zone of significant risk and halt before dissection before entering that zone. If we then consider the conditions around the gallbladder to be too dangerous, a safer method other than cholecystectomy should be chosen to finish the surgery. Consider laparoscopic subtotal cholecystectomy or cholecystostomy tube placement, and/or conversion to an open procedure based on the judgment of the attending surgeon.

Lastly, rule number 6 reminds us to always get help from another surgeon when the dissection or conditions are difficult.

In Europe, the SCP is not routinely taught during surgical training and formal education does not include it in its program. Therefore, the knowledge of the SCP among European surgeons comes from conferences, workshops or peer education. This paper was designed to assess the knowledge of the SCP among surgeons in Poland and Denmark and to get their opinion on the usefulness of the SCP rules in the everyday OR setting. The study was approved by the Ethical Committee of the Jagiellonian University and was registered in the ClinicalTrials.gov server, with ID: NCT03155321.

\section{Aim}

The aim of the study was to assess the knowledge of the SAGES Safe Cholecystectomy Program among surgeons in Poland and in Denmark. Moreover, the study was designed to gather information on the usefulness of SCP rules from the viewpoint of the surgeon. Such information is crucial in undertaking actions to further propagate and implement the program, for good guidelines should not only be medically and professionally intact, but also make the surgeon feel like they understand and appreciate the need to implement the guidelines.

\section{Material and methods}

The data were obtained via anonymous questionnaires distributed among participants of various national surgical conferences in Denmark and in Poland. The questionnaire consisted of three parts. The first part focused on demographic data with questions on age, surgical experience, gender, and professional status (resident vs. specialist). One control question was added to eliminate those who attend surgical conferences yet are not surgeons (mostly medical students or pharmaceutical company representatives). The second part asked whether the surgeon knows and uses the Tokyo Guidelines for the management of acute cholangitis and cholecystitis that were introduced in 2007 and updated in 2013. The next question asked about whether they know and use the SAGES Safe Cholecystectomy Program. The next four questions focused on the number of open and laparoscopic cholecystectomies performed, the occurrence of BDI and the occurrence of peritonitis. In the third part, the six rules of the SAGES SCP were listed and with each rule, the surgeon was to give their opinion on the usefulness of the rule on a numeric scale. It was a 10-point scale with 0 meaning that the rule is useless and 9 meaning that the rule is crucial during laparoscopic cholecystectomy.

In Denmark the study was performed on two separate occasions: at the Annual Symposium on Surgical Treatment of Gallstones (SSTG) in the Central Denmark Region and at the annual meeting of the Danish Surgical Society (DKS). In Poland, the same questionnaire was handed out at the Annual Practical Medicine Surgical Conference held in Krakow. The questionnaires were gathered in classic paper form.

\section{Statistical analysis}

The results were gathered and statistical analysis was performed comparing the results of two groups: residents and specialists. Descriptive statistics were used for quantitative analysis. Mean and standard deviation statistics were used where appropriate. Continuous variables were compared with the $t$-test or Wilcoxon rank sum test, depending on the normal distribution. Categorical variables were compared using the $\chi^{2}$ test.

\section{Results}

In Denmark, 84\% of the questionnaires handed out at the SSTG were filled out completely and $40 \%$ 
at the DKS Meeting. In total, 94 forms were obtained -47 (50\%) from surgical residents and 47 (50\%) from general surgery specialists. In Poland, 90 fully completed questionnaires were obtained, 23 (26\%) from residents and 67 (74\%) by specialists. Altogether, 184 filled forms were gathered, 114 (62\%) from surgical specialists and 70 (38\%) from residents (Figure 2). The basic demographic data are displayed in Table I. The mean age was $50 \pm 9.64$ years among specialists and $33.5 \pm 3.79$ years among residents. Eighty-three (72.8\%) of the specialists were male and $31(27.2 \%)$ female. The gender distribution was significantly different among residents, with 39 (55.7\%) male and 31 (44.3\%) female respondents. Naturally, the mean experience among specialists was significantly greater than among residents ( 22 vs. 4.6 years, $p<0.001$ ). In terms of number of laparoscopic cholecystectomies performed, $37.1 \%$ of the residents performed less than 50 and $62.9 \%$ between 50 and 500 procedures. None of the residents reported to have performed more than 500 laparoscopic cholecystectomies. Not surprisingly, specialists reported to have performed more procedures; $67.5 \%$ performed $50-500$ operations and $27.2 \%$ reported more than 500 operations performed. Most interestingly, only $21.4 \%$ of residents and $42.1 \%$ of specialists reported being aware of the SAGES SCP and using it on a clinical basis. Although statistical significance was obtained $(p=$ $0.004)$, the numbers are relatively low in both groups.

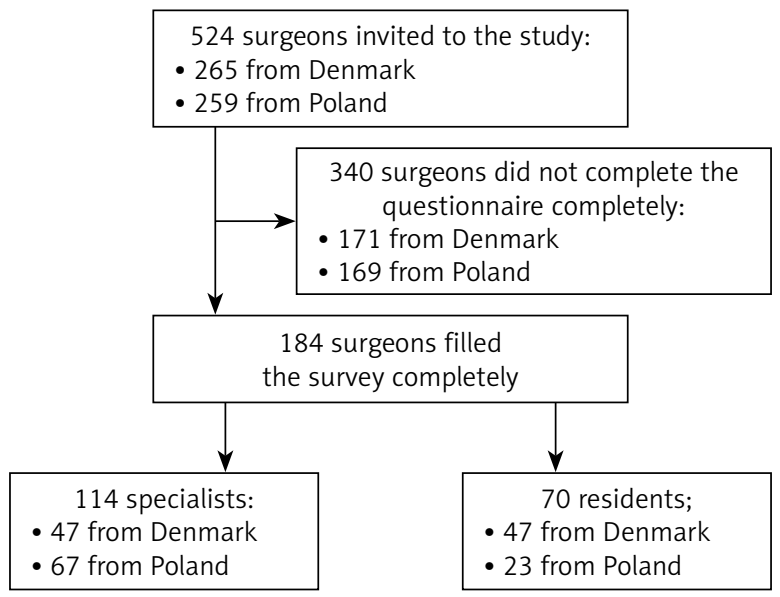

Figure 2. Patient selection process

Moreover, significantly more specialists have caused BDI during surgery compared to residents ( $40.4 \%$ vs. $11.4 \%, p<0.001$ ).

General opinion on the usefulness of each of the six SAGES SCP rules was high - the mean scores for each rule is above 6 points (out of 9). Detailed information on the results of the usefulness score are presented in Table II. A statistically significant difference in the opinions of usefulness of the rules between residents and specialists was observed in regard to three rules: rules number 2 and 6 were considered more important by residents (7.07 vs. 6.01 , $p=0.025$ and 8.70 vs. $8.27, p<0.001$ respectively),

Table I. Characteristics of the groups

\begin{tabular}{|c|c|c|c|}
\hline Parameter & Residents $(n=70)$ & Specialists $(n=114)$ & $P$-value \\
\hline Age, mean [years] & $33.5 \pm 3.79$ & $50 \pm 9.64$ & $<0.001$ \\
\hline Gender: & & & $<0.001$ \\
\hline Male & $39(55.7 \%)$ & $83(72.8 \%)$ & \\
\hline Female & $31(44.3 \%)$ & $31(27.2 \%)$ & \\
\hline Experience [years] & $4.6 \pm 2.6$ & $22 \pm 10.6$ & $<0.001$ \\
\hline Know the Updated Tokyo Guidelines & $17(24.3 \%)$ & $33(28.9 \%)$ & 0.609 \\
\hline Know the SAGES SCP & $15(21.4 \%)$ & $48(42.1 \%)$ & 0.004 \\
\hline \multicolumn{4}{|c|}{ Number of procedures performed laparoscopically: } \\
\hline$<50$ & $26(37.1 \%)$ & $6(5.3 \%)$ & $<0.001$ \\
\hline $50-500$ & $44(62.9 \%)$ & $77(67.5 \%)$ & 0.526 \\
\hline$>500$ & $0(0 \%)$ & $31(27.2 \%)$ & $<0.001$ \\
\hline Ever caused BDI injury & $8(11.4 \%)$ & $46(40.4 \%)$ & $<0.001$ \\
\hline Ever caused choleperitonitis & $13(18.6 \%)$ & $62(54.4 \%)$ & $<0.001$ \\
\hline
\end{tabular}

SCP - Safe Cholecystectomy Program, BDI - bile duct injury. 
Table II. Usefulness score of each of the SAGES SCP Rules

\begin{tabular}{|lccc|}
\hline Variable & $\begin{array}{c}\text { Residents }(n=70) \\
\text { Mean (min.-max., SD) }\end{array}$ & $\begin{array}{c}\text { Specialists }(n=114) \\
\text { Mean (min.-max., SD) }\end{array}$ & $P$-value \\
\hline $\begin{array}{l}\text { Rule 1a (Clear the hepatocystic triangle of fat and } \\
\text { fibrous tissue) }\end{array}$ & $7.93(2-9,1.58)$ & $8.06(1-9,1.44)$ & 0.632 \\
\hline Rule 1b (Expose cystic plate) & $7.06(1-9,2.24)$ & $6.86(1-9,2.12)$ & 0.605 \\
\hline $\begin{array}{l}\text { Rule 1c (Only two structures are entering the } \\
\text { gallbladder) }\end{array}$ & $6.76(2-9,2.88)$ & $7.14(1-9,2.69)$ & 0.121 \\
\hline Rule 2 (Consider intra-operative time-out) & $7.07(1-9,2.26)$ & $\mathbf{6 . 0 1}(1-9,2.71)$ & $\mathbf{0 . 0 2 5}$ \\
\hline Rule 3 (Understand potential for aberrant anatomy) & $\mathbf{8 . 3 6}(3-9,1.19)$ & $\mathbf{8 . 7 3 ( 5 - 9 , 0 . 6 9 )}$ & $<0.001$ \\
\hline Rule 4 (Make liberal use of cholangiography) & $7.27(2-9,2.12)$ & $7.26(1-9,2.19)$ & 0.820 \\
\hline Rule 5 (Recognize dangerous zone) & $8.02(3-9,1.30)$ & $8.32(3-9,1.08)$ & 0.230 \\
\hline Rule 6 (Get help from another surgeon) & $\mathbf{8 . 7 0 ( 6 - 9 , 0 . 6 4 )}$ & $\mathbf{8 . 2 7}(1-9,1.59)$ & $<0.001$ \\
\hline
\end{tabular}

Values in table present mean scores. Min score is 0 , max score is 9. Statistically significant differences are in bold.

and rule number 3 was considered more important by specialists ( 8.73 vs. $8.36, p<0.001)$.

\section{Discussion}

Bile duct injuries remain a big concern in the era of laparoscopic cholecystectomy. Currently, over $88 \%$ of all cholecystectomies in the USA are performed laparoscopically, and the number of open cholecystectomies will probably further decrease with time. Although the incidence of BDI has fallen during the first decade after implementation of laparoscopic technique, it seems to have plateaued at a level that still exceeds that of open cholecystectomy [5]. There are publications that report BDI after laparoscopic to be as low as $0.08 \%$ [9]; however, the majority of publications report the incidence to be somewhat higher, with numbers fluctuating from $0.3 \%[10,11]$ to as high as $2.6 \%$ [12]. A systematic review consisting of 46 studies and a total of 2626 patients reported the incidence of $\mathrm{BDI}$ at a rate of $0.72 \%$ in patients with no acute cholecystitis [13]. On the other hand, a retrospective Finnish study on a large cohort proved that OC was associated with a higher BDI incidence rate than $L C$, although the injury to the bile duct was minor in most of the OC cases, and BDI after LC tended to be more severe [14]. Such a wide range of reported BDI incidence is perplexing and may be due to difficulties in categorizing and reporting of BDI. Many BDI classification schemes have been published (e.g. Bismuth, Strasber, EAES [15, 16]), yet not one of them has been widely adopted and used as a standard for reporting of BDI, which causes room for bias in comparing different studies and reports. Regardless of the differences in incidence data, the high frequency of this surgical procedure makes BDI a significant burden on healthcare systems, with BDI being a costly complication to treat. It is therefore advisable to seek ways to further reduce the incidence of BDI after laparoscopic cholecystectomy. SAGES has developed a Culture of Safety Program implementing a set of rules to follow during an operation, one of which is the Critical View of Safety that is to be achieved in each cholecystectomy. This has been widely promoted in the US, yet is still not widespread among surgeons in Europe. In 2012, the EAES published clinical practice guidelines on the prevention and treatment of bile duct injuries during laparoscopic cholecystectomy [16], yet no initiative comparable to the SCP has emerged. The reluctance of adopting the Safe Cholecystectomy Program (SCP) may stem from the fact that there has been research showing that the SCP has not proven to have a positive effect on reduction of CBDI after $L C$ in countries where the documentation of CVS is mandatory [17]. A different study concluded that there is no correlation of CVS with prevention of biliary injury [18]. Other studies have however reported a significant drop in the BDI incidence, equaling that of OC, after implementing routine CVS documentation during LC [9].

In this study we not only wanted to assess the knowledge of the SAGES SCP among surgeons in Denmark and in Poland. The study itself had an educational value to it, giving a detailed description of the SCP rules and clear education on how to achieve the CVS. The reach of the project was not high, but in Denmark 
almost $20 \%$ of all registered surgeons took part in the study, hence revising or learning about the SCP.

The results have shown that the knowledge of the SCP among European surgeons is low: only $21.4 \%$ of residents and $42.1 \%$ of specialists stated that they know and implement the SAGES SCP in the clinical setting. The SCP is not included in the program of formal education of surgeons in most European countries. The fact that twice as many specialists have heard of it may stem from the fact that they have attended more surgical conferences or are more acquainted with the literature. It is clear that there still is room for improvement. The fact that over $40 \%$ of specialists and $11 \%$ of residents stated that they have caused BDI during LC is surprising and raises the question of what their understanding of BDI is, as these numbers seem relatively high, bearing in mind the low incidence of BDI.

It is promising that the general score of usefulness of the SAGES SCP Rules was high; none of the rules was rated lower than 6 on a 10-point scale (0 meaning totally useless, 9 meaning absolutely crucial). The mean usefulness score for most of the rules was around 8 points (see Table II for details). Differences between residents and specialists were noted in three of the rules; rule no 2 was rated significantly more important by residents than specialists (7.07 vs $6.01, p=0.025)$. This rule indicated that an intra-operative time-out should be considered before clipping, cutting or dissecting any ductal structures. Specialists are less likely to pause during the operation, probably because they have fewer doubts about the next step. This, however, may lead to complications, as rushing during surgery is never safe. Residents take their time, especially during important moments in surgery, and are more likely to pause and re-think their approach before cutting a ductal structure.

Moreover, residents have reported rule no. 6 to be more important in comparison with specialists (8.70 vs. $8.27, p<0.001)$ - this rule suggests getting help from another surgeon when the dissection or conditions are difficult. It is not surprising that it was rated higher by residents, as they are naturally encouraged to seek help from their more experienced colleagues as a natural process of education. Although the usefulness score from specialists was lower, it was still very high, with an average score of 8.27 , which is a positive sign. Last but no least, rule no. 3 was considered more important by specialists $(8.73$ vs. $8.36, p<0.001)$. This rule highlights the need to understand the potential for aberrant anatomy in cases. This may suggest that residents, although aware of anatomical differences, are less proficient in determining the actual anatomy of bile ducts and vessels in each of the cases and may find it more challenging to correctly identify anatomical differences if present. More stress should be placed on educating about different variations of bile-tree and vascular anatomy during surgical training.

The results of the study show that surgeons are accepting of the SCP and willing to use it in everyday practice. However, the literature in this matter suggests otherwise. The CVS technique is not unique in identifying the anatomy of the cystic duct and the cystic artery during surgery. With the infundibular technique, top-down technique and routine cholangiography, the CVS still remains an option for the surgeon, not a necessity. Furthermore, the reluctance in adopting new techniques among surgeons is high, and the low incidence of BDI during LC makes it even harder for surgeons to find motivation to change their habits [19]. Interestingly, a study showed that even in hospitals in which the CVS is routinely used and operative notes report that the CVS had been obtained, about $25 \%$ of those who claimed to have obtained it did so inadequately [20]. This suggests that surgeons may not fully comprehend the details and the rationale behind the CVS and may for example confuse it with the infundibular technique [21].

A different study looked at video transmissions of laparoscopic cholecystectomies available online, as they very often are a source of education or case preparation. The researchers analyzed 160 videos of laparoscopic cholecystectomy posted on YouTube and found that the CVS was achieved in only one video, whereas in the remaining 159 videos the rules of CVS were not met [22]. This is concerning given the fact that for many surgeons video transmissions are the leading source of education, and open video portals such as YouTube are very often the go-to sites where surgeons search for videos. Yet the quality of that material is very often questionable. It is therefore recommended to seek educational videos on medical websites such as the SAGES or WebSurg websites.

One should also remember that in acute cases, in which patients are unfit for surgery, less invasive methods of treatment are available. In recent years, endoscopic ultrasound-guided gallbladder drainage (EUSGBD) has become a popular method of treatment of such patients. It proves to be an effective and safe 
method of treatment in patients with acute cholecystitis who are unsuitable for cholecystectomy [23].

The need to further educate surgeons in techniques of laparoscopic cholecystectomy is undoubted - this procedure will probably completely replace the open cholecystectomy surgery, not only because of better treatment outcomes, fewer complications and shorter hospital stay. It has been proven that the laparoscopic procedure is more cost effective for the payer, despite the costly equipment that needs to be used for the surgery. A recent publication by Smigielski et al. presented a precise financial comparison of these two procedures [24].

\section{Conclusions}

Overall, the SAGES Safe Cholecystectomy Program was positively assessed by the respondents and the results of the study give a clear indication that further educational programs should be implemented to broaden the knowledge of the SCP among European surgeons in order to continuously strive towards minimizing the risk of BDI during laparoscopic cholecystectomy. It seems reasonable that the SCP should be adopted into the official surgical residency training in Europe.

\section{Conflict of interest}

The authors declare no conflict of interest.

\section{References}

1. McKinley SK, Brunt LM, Schwaitzberg SD. Prevention of bile duct injury: the case for incorporating educational theories of expertise. Surg Endosc 2014; 28: 3385-91.

2. Ingraham AM, Cohen ME, Ko CY, et al. A current profile and assessment of North American cholecystectomy: results from the American College of Surgeons National Surgical Quality Improvement Program. J Am Coll Surg 2010; 211: 176-86.

3. Strasberg SM, Brunt LM. Rationale and use of the critical view of safety in laparoscopic cholecystectomy. J Am Coll Surg 2010; 211: 132-8.

4. Barrett M, Asbun HJ, Chien HL, et al. Bile duct injury and morbidity following cholecystectomy: a need for improvement. Surg Endosc 2018; 32: 1683-8.

5. Ahrendt SA, Pitt HA. Surgical therapy of iatrogenic lesions of biliary tract. World I Surg 2001; 25: 1360-5.

6. Avgerinos C, Kelgiorgi D, Touloumis Z, et al. One thousand laparoscopic cholecystectomies in a single surgical unit using the "critical view of safety" technique. J Gastrointest Surg 2009; 13: 498-503.

7. Heistermann HP, Tobusch A, Palmes D. Prevention of bile duct injuries after laparoscopic cholecystectomy. "The critical view of safety". Zentralbl Chir 2006; 131: 460-5.
8. Yegiyants S, Collins JC. Operative strategy can reduce the incidence of major bile duct injury in laparoscopic cholecystectomy. Am Surg 2008; 74: 985-7.

9. Halbert C, Pagkratis S, Yang J, et al. Beyond the learning curve: incidence of bile duct injuries following laparoscopic cholecystectomy normalize to open in the modern era. Surg Endosc 2016; 30: 2239-43.

10. Yamashita Y, Kimura T, Matsumoto S. A safe laparoscopic cholecystectomy depends upon the establishment of a critical view of safety. Surgery Today 2010; 40: 507-13.

11. SAGES (2014) The sages safe cholecystectomy program. http:// www.sages.org/safe-cholecystectomy-program/10.

12. Aziz H, Pandit V, Joseph B, et al. Age and obesity are independent predictors of bile duct injuries in patients undergoing laparoscopic cholecystectomy. World J Surg 2015; 39: 1804-8.

13. Berthou J, Dron B, Charbonneau P, et al. Evaluation of laparoscopic treatment of common bile duct stones in a prospective series of 505 patients: indications and results. Surg Endosc 2007; 21: 1970-4.

14. Bingham J, McKie LD, McLoughlin J. Biliary complications associated with laparoscopic cholecystectomy - an analysis of common misconceptions. Ulster Med J 2000; 69: 106-11.

15. Chun K. Recent classifications of the common bile duct injury. Korean J Hepatobiliary Pancreat Surg 2014; 18: 69-72.

16. Eikermann M, Siegel R, Broeders I, et al. Prevention and treatment of bile duct injuries during laparoscopic cholecystectomy: the clinical practice guidelines of the European Association for Endoscopic Surgery (EAES). Surg Endosc 2012; 26: 3003-39.

17. Sanford DE, Strasberg SM. A simple effective method for generation of a permanent record of the critical view of safety during laparoscopic cholecystectomy by intraoperative "doublet" photography. J Am Coll Surg 2014; 218: 170-8.

18. Kohn JF, Trenk A, Kuchta K, et al. Characterization of common bile duct injury after laparoscopic cholecystectomy in a high-volume hospital system. Surg Endosc 2018; 32: 1184-91.

19. Daly SC, Deziel DJ, Li X, et al. Current practices in biliary surgery: do we practice what we teach? Surg Endosc 2016; 30: 3345-50.

20. Stefanidis D, Chintalapudi N, Anderson-Montoya B, et al. How often do surgeons obtain the critical view of safety during laparoscopic cholecystectomy? Surg Endosc 2017; 31: 142-6.

21. Chen CB, Palazzo F, Doane SM, et al. Increasing resident utilization and recognition of the critical view of safety during laparoscopic cholecystectomy: a pilot study from an Academic Medical Center. Surg Endosc 2017; 31: 1627-35.

22. Deal SB, Alseidi AA. Concerns of quality and safety in public domain surgical education videos: an assessment of the critical view of safety in frequently used laparoscopic cholecystectomy videos. J Am Coll Surg 2017; 225: 725-30.

23. Sowier S, Sowier A, Wiechowska-Kozłowska A, et al. Initial experience with endoscopic ultrasound-guided gallbladder drainage. Videosurgery Miniinv 2018. https://doi.org/10.5114/ wiitm.2018.79528.

24. Śmigielski J, Piskorz Ł, Koptas W. Comparison of treatment costs of laparoscopic and open surgery. Videosurgery Miniinv 2015; 10: 437-41.

Received: 3.01.2019, accepted: 11.02.2019. 